\title{
Frailty in Myocardial Infarction Patients: A Paradigm Shift
}

\author{
Mariam Hassan ${ }^{1}$, Hanan Sheikh Ibrahim ${ }^{2}$, Samer Ellahham ${ }^{2,3}$ \\ ${ }^{1}$ Gulf Medical University, Ajman, United Arab Emirates \\ ${ }^{2}$ Cleveland Clinic Abu Dhabi and Cleveland Clinic Foundation, Abu Dhabi, United Arab Emirates \\ ${ }^{3}$ Cleveland Clinic Foundation, Ohio, United States of America
}

\begin{abstract}
An insightful review of evidence-based literature unveils a drastically increasing incidence of myocardial infarction (MI). As the global population becomes older, the frequency of adverse coronary events escalates proportionally. The modifiable and non-modifiable risk factors that contribute toward the development of MI have been enumerated, and the aftereffects which may follow are known to be even more detrimental. Nevertheless, it is imperative to acknowledge that the management of MI patients does not only surround their cardiovascular health but is governed by many other seemingly superfluous concerns - some of which fall under the umbrella of frailty. A coalesce of frailty identification and appreciation of its subsequent role in prognostication and management is revolutionary. This further proves the need for considerable collaboration between geriatricians that meticulously deliberate the quintessential all-round care of the elderly as well as the cardiologists whose aim is to optimize cardiac function, survival and quality of life. Thus, the purpose of this mini-review is to ponder upon and adopt a validated assessment tool for the purposes of diagnosing frailty and prognostication amongst MI. This will allow for purposeful formulation of individualized management plans that could reduce the rates of mounting morbidities and all-cause mortality.
\end{abstract}

Keywords: Acute coronary event; Cardiovascular health; CGA; Elderly; Frailty; Geriatric; Identification; Mortality; Myocardial infarction; Risk factors

\section{ABBREVIATIONS}

ADL: Activities of Daily Living; GFI: Groningen Frailty Indicator; CFS: Clinical Frailty Scale; MI: Myocardial Infarction; CGA: Comprehensive Geriatric Assessment; NHS: National Health Service; CHS: Cardiovascular Health Study; NSTEMI: Non-ST Elevation Myocardial Infarction; EFS: Edmonton Frail Scale; STEMI: ST Elevation Myocardial Infarction; FI-CD: Frailty Index-Cumulative Deficits; TIMI: Thrombolysis in Myocardial Infarction; FP: Frailty Phenotype; WHO: World Health Organization

\section{INTRODUCTION}

The World Population Prospects (2017) discloses the proportion of the global population aged 60 years or older-comprising an estimate of 13 per cent. Extrapolated data anticipates this figure to reach 21.3 per cent in 2050 [1]. The affliction of cardiovascular health parallels that of an expanding elderly population. A myocardial infarction (MI), for instance, cultivates a substantial variety of comorbid complications which necessitates patient-centered and devoted quality care despite paramount healthcare expenditure and a pending socioeconomic decline.

International guidelines recommend that myocardial injury is classified according to the etiology. It can be a result of plaque rupture or a deficit in oxygen supply to myocardial tissue that is secondary to a precedent illness. Regardless, the modifiable risk factors should be recognized and dealt with without further delay. The American Geriatrics Society highlights four major

${ }^{*}$ Correspondence to: Dr. Samer Ellahham, Cleveland Clinic Abu Dhabi and Cleveland Clinic Foundation, UAE/CCF 1950 Richmond Road, TR303, Lyndhurst, OH 44124, Abu Dhabi, UAE, Tel: 971508113142; E-mail: ellahas@clevelandclinicabudhabi.ae

Received: April 25, 2019; Accepted: May 17, 2019; Published: May 24, 2019

Citation: Ellahham S, Ibrahim HS, Hassan M (2019) Frailty in Myocardial Infarction Patients: A Paradigm Shift. Cardiovasc Pharm Open Access. 8: 254. doi: 10.35248/2329-6607.19.8.254

Copyright: (C) 2019 Ellahham S, et al. This is an open access article distributed under the terms of the Creative Commons Attribution License, which permits unrestricted use, distribution, and reproduction in any medium, provided the original work is properly cited. 
predisposing factors of cardiovascular disease: hypertension, diabetes mellitus, dyslipidemia and smoking [2]. Other predisposing variables include a history of coronary artery disease, sedentary lifestyle, excessive alcohol ingestion and being engorged in psychosocial stress. While these are tackled with prescribing an appropriate array of medications as well as counselling on avoiding high-risk diets and engaging in adequate exercise, the real challenge lies in persuading older individuals that present with ancillary obstacles.

The obstacles referred to here fall under the spectrum of frailty. The Thrombolysis in Myocardial Infarction (TIMI) risk score estimates mortality in patients with unstable angina or non-ST elevation MI (NSTEMI), and the HEART score predicts 6-week risk of adverse cardiac event [3,4]. Yet, neither one probes into the daily hindrances of a patient which impair the path towards stable recovery. If a patient were to be investigated for a frail status through a constructive collaboration between cardiologists and geriatricians, then a truly comprehensive evaluation has been performed. A review of literature reaffirms the unequivocal nature of frailty and the lack of a standardized scoring system which will signify a frail patient. For this reason, an assessment tool that is internationally recognized, easily accessible, and simple in presentation and use should be highly sought after.

Because the heart of geriatric practice is becoming aptly integrated into the field of cardiology, this mini-review attempts to delineate the concept of frailty with a methodical outline of the various assessment tools out there.

\section{Myocardial infarction: A global threat}

According to the World Health Organization (WHO), "there are 32.4 million myocardial infarctions and strokes worldwide every year [5]." The incidence of acute coronary events reflects the rise in age which has been observed in general populations; "20\% of patients aged over 65 years receive percutaneous coronary interventions and $27 \%$ of patients aged over 70 years receiving coronary angiography [6]." It so happens that all of these patients exhibit the typical configuration of frailty.

Frailty shall be elaborated upon in the forthcoming segments. For now, it will suffice to apprehend that a frail status implies a biological age superseding the respective chronological age. 'Insights from the CONCORDANCE Registry' emphasizes that, in patients with significant cardiovascular disease, the prevalence of frailty "may be as high as 60\% [7]." Frailty is associated with worst cardiac outcomes after an MI [7]. These patients are at an "increased risk of recurrent infarctions and have an annual death rate of 5 per cent" or "six times that in people of the same age who do not have coronary heart disease [5]." It is now clear that frailty may pose as an independent risk factor for mortality. Yet, it should be noted that the relationship between MI and frailty is a bidirectional one. Frail subjects are also more likely to present with a coronary event. Similarly, patients with cardiovascular disease are more likely to have a "functional impairment" alluding to a frail status [6].

Although a great deal of effort and advancements have been made to allow for dissolution of an MI and reduce likelihood of recurrence or complications, it is still a major vehicle for all- cause mortality. An acute coronary syndrome alone imposes a great deal of stress on the human body [6]. Even so, studies show that cardiac-related risk factors are not sufficient to explain neither the development, progression nor adverse effects of MI. Frail individuals have "longer lengths of hospital stay and are more likely to be readmitted with recurrent cardiovascular events [6]."

\section{Recognizing frailty}

The concept of frailty is being exponentially explored for the purposes of determining disease etiology, estimating prevalence, risk assessment, and as a guide for clinical decision-making and target intervention in cardiology, oncology, surgery, orthopedics, organ transplantation, trauma and possibly in other medical subspecialties [8]. It is also, however, becoming progressively perplexing to adopt an assessment tool which clearly reflects the true meaning of frailty and encompasses all the risk factors that are determinants of an accurate diagnosis. A patient may segue into and qualify for the frailty spectrum by any disturbance in Activities of Daily Living (ADL), for example. Therefore, it is imperative to note that there are a multitude of trivialized elements which should be identified and indisputably held accountable for frailty in everyday clinical practice.

In order to attain such consensus, a standardized description of the term 'frailty' will be utilized. Frailty, as described in 'Frailty syndrome: an overview' (2014), is an "age-associated decline in physiologic reserve and function across multiple organ systems," resulting in diminished strength and endurance, increased vulnerability to stressors, risk of falls, disability, hospitalization, and mortality [9]. This can be easily confused with aging whereby there is "deterioration of functional properties at the cellular, tissue and organ level [10]." Frailty and aging are both associated with disrupted homeostasis. The key difference is that, with aging, the homeodynamic failure is global. Frailty exhibits an exacerbating "energy metabolism and neuromuscular changes" instead [10]. Moreover, the biomarker distribution in the frail population is distinct from the corresponding agerelated changes observed in the non-frail or "normal" individuals [10].

The operationalization of frailty permits a comprehensive investigation into the pillars of frailty, recognition of the most suitable assessment tool, followed by a focused application on a largely heterogenous population of patients presenting with, for example, cardiac-related conditions. As the elderly population multiples, the incidence of frailty and acute coronary syndromes foster. It is only reasonable that cardiologists employ the visions of a geriatrician whose primary concern lies in tackling frailty. In doing so, the management essentially becomes tailored to the patient's personal needs and likens to their overall wellbeing.

\section{Role of frailty in alleviating disease burden}

The potential ramifications of an acute MI patient include, but are not limited to, an arrhythmia, heart failure, recurrent MI, angina, stroke and death [11]. These outcomes are known and well-established. The relationship between acute coronary disease and frailty showcases a bidirectional configuration [12]. Frailty is reported to be more common in patients presenting 
with a cardiovascular disease-irrespective of whether frailty was an instigator of this incident or a mere result of it.

In the recent years, the repercussions of frailty upon coronary vascular disease has become heavily investigated. A study with 307 NSTEMI patients aged over 75 years divulged that $48.5 \%$ were frail and "experienced increased rates of re-infarction, revascularization, hospitalization, major bleeding, stroke and need for dialysis and mortality compared with non-frail [13]." Furthermore, the National Health Service (NHS) has found that "frailty and prefrailty are associated with increased risk of death [14]." Consequently, accentuating the association between a prefrail status patient and mortality would be the first step in "identifying potential targets of intervention in future studies [14]."

If we are to study the impact of frailty upon myocardial infarction patients, the methodology must address the basis of geriatric practice as well as be pertinent to a cardiology care unit. A unifying overlap between geriatricians and cardiologists will help construct a scheme that not only is apprehensive about the patient's medical liability but aspires to achieve the best quality of life possible.

There will certainly remain a degree of subjectivity and ambiguity in coming to terms with a definitive discernment of what clinicians deem frail. Regardless, a demarcated estimation is still more substantial than paying no attention to such an intricate and multifaceted risk factor. In addition, while validity of the proposed tool remains of utmost priority, meaning that it effectively distinguishes a frail patient with taking their heart problem into consideration, its feasibility is undoubtedly fundamental. Clinicians should be able to promptly ascertain whether a patient meets the criteria of frail and tailor their management plan accordingly.

\section{Frailty assessment tools}

The Comprehensive Geriatric Assessment (CGA) is a multidisciplinary diagnostic measure that ensures an effective and systematic evaluation of patients liable to frailty, thereby aiming to decrease the chances of imminent morbidity [15]. It is widely used in the inpatient - and to a lesser extent, outpatientsetting to evaluate the core components of frailty, including functional capacity, fall risk, cognition, mood, polypharmacy, social support, financial concerns, goals of care and advance care preferences [15]. It also deliberates nutrition, weight change, urinary continence, sexual function, vision, hearing, dentition, living situation and spirituality [15].

A study in Sweden concluded that "acute care in a CGA unit with direct admission was associated with higher levels of patient satisfaction compared with conventional acute care via the emergency room [16]." Another study concerning the effectiveness of CGA in elderly patients with coronary heart disease highlights that this approach "adds substantial information to evaluate the clinical, psychological and functional aspects in elderly CHD patients" which further improves "living quality, positive mental attitude" as well as "reducing the hospital days and cost [17]." Thus, an assessment tool which covers the domains of the CGA would be close to ideal.

The Rapid Geriatric Assessment (RGA) takes less time for completion, and questions pertaining to a frail phenotype are exhibited in a succinct, user-friendly interface. RGA incorporates several frailty elements that are vital to ascertain as a part of the evaluation process [18]. However, the RGA overlooks many other factors including mood, polypharmacy, social frailty and finance. Social frailty, for example, is highly underestimated and could be the cause of substantial vulnerability which ultimately leads to frailty. Polypharmacy is yet another potential catalyst towards the development of a frail clinical picture. The tendency to neglect polypharmacy, although infrequent, is perilous. It has been shown to potentiate fall risk, and such inclination suggests that polypharmacy denotes frailty, or can instigate a frail state brought about by a single fall.

Fried's Frailty Phenotype (FP) has been derived from the Cardiovascular Health Study (CHS) Index. Despite its widespread use in the geriatric cardiology sector, it is deemed as unidirectional in terms of its simplicity. The results, although reproducible, lack insight into cognition, mood and the social frailty domains. Many risk factors that comprise frailty are not addressed here. It focuses especially on the physical aspects of a patient in terms of endurance, physical activity, energy levels, walking speed and weight loss [19].

The constituents of the FRAIL Index are limited to Fatigue, Resistance, Ambulation, Illness and Loss of Weight. Similarly, the Vulnerable Elders Survey-13 and PRISMA-7 do not cover the important domains of the CGA [20,21].

The Clinical Frailty Scale (CFS) has been "validated as an adverse outcome predictor in older people that have been hospitalized [22]." The disadvantage here is that the scoring of frailty is subjective as the health professional must determine the description which best fits the patient after having done a CGA. Ultimately, the aforementioned diagnostic tools inquire about a select few domains of the CGA-limiting our capability to appreciate the true magnitude of frailty.

The Frailty Index (FI) of Cumulative Deficits (FI-CD), derived from the Canadian Study of Health and Aging, was first proposed by Rockwood and Mitnitski [22]. It compiles 70 deficits, and those applicable the patient are then divided by the total [22]. A ratio of 0.25 or more means that the patient qualifies as frail. This is a tedious process for a clinician whom must urgently decipher whether the patient is frail or not. The assessment tool entails ruling in or out every item as each one counts for a single point.

The Groningen Frailty Indicator (GFI) has demonstrated high internal consistency, construct and clinical validity [23]. The questions are straightforward - demanding 'Yes' or 'No' answers, too. It is unsophisticated in presentation leading to less time-consumption. Conversely, the predictive power is reportedly poor [24].

The Edmonton Frail Scale (EFS) is a brief, user-friendly screening interview with "acceptable internal consistency, good construct validity" and reliability [25]. EFS is cheap, convenient 
and has been utilized by non-specialists who have no formal training in geriatric care proving its potential as a practical measure of frailty in a variety of settings, like acute care [26]. In spite of this, the EFS requires that an elderly patient performs tasks to sufficiently evaluate the cognition and functional performance domains. This is unlikely to be completed in neither an acute MI nor recovery patient.

Tilburg Frailty Indicator (TFI) delves into the determinants of frailty, its physical, psychological and social components. A recent systematic review concluded that there is "robust evidence of this user-friendly questionnaire's reliability, validity and quick and easy administration [27].” It primarily consists of 'Yes' or 'No' questions - all of which can be swiftly answered with the exception of net monthly household income. This will require modification as the currency and listed values would not be applicable globally. On the other hand, "overviews of several instruments" have suggested that the TFI is "not the best predictor of all adverse outcomes of frailty [27]." Consequently, it has been suggested that further studies are needed "to establish whether the TFI is suitable for intervention studies...for specific target groups [27].”

\section{Proposal for frailty in myocardial infarction}

The frailty assessment tools which have been notably utilized amongst cardiologists include Fried's FP and gait speed, which are common in studies of CVD. The CFS has also been stressed upon with regards to cardiac intervention due to its graded scale from very fit to severely frail. However, if the focus were to be narrowed on patients having been admitted for an acute MI, Fried's FP, FI-CD and the TFI have been employed for recognition of a frail status patient and combating the likelihood of an impending adverse outcome. Because we do not have one internationally recognized and standardized diagnostic tool for frailty, there is an ongoing debate with regards to which is more likely to be incorporated into clinical practice. Nonetheless, a thorough rundown of the available frailty surveys, followed by a logical selection to the most clinically applicable tool, will help determine the prognostic implications of frailty upon the selected group of individuals-namely MI patients.

The authors propose that two of the said frailty measures be appraised: EFS and TFI. There are many advantages seen with the use of the EFS. The notable and perhaps most encouraging reasons are: it requires less than 5 minutes to administer, has been customized for non-geriatricians, and helps stratify "the state of fragility in a group of institutionalized elderly [28]." The EFS has proven to be associated with other geriatric conditions like "mood, mental, functional and nutritional status [28]." It can also be modified in such a way that it eliminates the performance of tasks in the cognition and functional performance domains.

The TFI is a self-report instrument with low caregiver costs. Likewise, an inquiry into household income need not involve figures that may be considered invasive to the patient. The elimination of figures will allow it to instantly become easier to respond to and more relevant to global citizens. This would also present as an opportunity to confirm its suitability for the target group of interest, being acute MI patients.

\section{CONCLUSION}

The incidence of acute coronary disease is expected to surge in the coming years with an ever-growing elderly population. Whether this occurrence provokes the steadfast onset of a clinically frail picture, or is secondary to an innate reduction in biologic reserve, cannot always be distinguished. Regardless, modifiable cardiovascular risks must be put to a halt and a detailed analysis of the elements encompassing frailty should be revised.

Frailty is a rapidly emerging, innovative phenomenon that illuminates upon the human body's susceptibility to stressors that provoke devastating consequences. Since it has been introduced by Fried, a consensus on the most suitable definition has yet to be reached and so it is being rectified till this day. As such, it is not entirely understood by many clinicians whom encounter frail patients more often than expected. The criteria for frailty is poorly outlined and, in turn, the diagnosis is preferably made on a more subjective basis or disregarded entirely. This explains why the implications of frailty are being undermined in everyday clinical practice.

The geriatric gold standard for frailty recognition is the CGA, but this frailty tool is quite time-consuming and is largely dependent upon the environment as well as administers' capability and expertise to grasp the capacity for repercussions. An assessment tool that is derived from or closely resembles the CGA would serve the purpose of frailty identification, prognostication and management in an inpatient setting. Our aim is to stress upon the fact that we still lack a chosen frailty model that is ideal in its assessment of any elderly individual whom visits the emergency department or has been admitted to a cardiac care unit. What makes this process perplexing is that there is a considerable overlap in the various assessment tools, but it would only make sense that each one lends themselves to different predictive uses.

Additionally, a critical MI patient will require a swift assessment for risk factors that go beyond the scope of only cardiac. These factors must not be belittled as they could either encourage or hinder the road to recovery. We need to be ever more cautious in our diagnosis of frailty, and management can be just only if this independent risk factor has been probed into.

The take-home recommendation here is that initiatives should be directed towards an objective identification of frailtyprovoking variables, selection of the most practical and feasible measurement tool, and target application in specific contexts, such as in cardiovascular disease patients. We took a look over the most widely used assessment tools, and have come to the conclusion that the EFS or TFI could be further studied, modified and implemented to allow for use in an emergent scenario, like an acute MI.

In the meantime, one may ponder upon the extent to which a balance between validity and feasibility of an assessment tool can be achieved. Behavioral and pharmacologic support are the mainstay of cardiac care management, but a selection of a single 
uniformly agreed upon frailty assessment tool could be counterproductive in the long run. It may be argued that this is, in fact, contradictory to the aim of having tailored care for each patient.

\section{REFERENCES}

1. https://www.un.org/en/sections/issues-depth/ageing/.

2. https://geriatricscareonline.org/FullText/B030/ B030_VOL001_PART001_SEC006_CH048

3. Antman EM. The TIMI risk score for unstable angina/non-ST elevation MI: A method for prognostication and therapeutic decision making. 2000;284: 835-842.

4. Brady W. The HEART score: A guide to its application in the emergency department. Turkish J Emergency Medicine. 2018;18: $47-51$.

5. https://www.who.int/cardiovascular_diseases/priorities/ secondary_prevention/country/en/index1.html.

6. Walker D. Editor's Choice - Frailty and the management of patients with acute cardiovascular disease: A position paper from the Acute Cardiovascular Care Association. Eur Heart J: Acute Cardiovasc Care. 2018;7: 176-193.

7. Patel A. Frailty and Outcomes After Myocardial Infarction: Insights From the CONCORDANCE Registry | Journal of the American Heart Association. J Am Heart Ass. 2018;7.

8. https://www.americangeriatrics.org/sites/default/files/inlinefiles/ravi_varadhan.pdf

9. https://www.dovepress.com/frailty-syndrome-an-overview-peerreviewed-article-CIA

10. Fedarko N. The Biology of Aging and Frailty. Clin Geriatr Med. 2011;27: 27-37.

11. https://www.uptodate.com/contents/prognosis-after-myocardialinfarction

12. Kleipool E, Hoogendijk E. Frailty in Older Adults with Cardiovascular Disease: Cause, Effect or Both. Aging Dis. 2018;9: 489-497.

13. Ekerstad N. Frailty is independently associated with 1-year mortality for elderly patients with non-ST-segment elevation myocardial infarction. Eur J Prev Cardiol. 2014;21: 1216-1224.

14. Crow RS, Lohman MC, Titus AJ, Bruce ML, Mackenzie TA, Bartels SJ, et al. Mortality Risk Along the Frailty Spectrum: Data from the National Health and Nutrition Examination Survey 1999 to 2004 . 2018;66:496-502.
15. https://www.uptodate.com/contents/comprehensive-geriatricassessment.

16. Ekerstad N, Östberg G. Are frail elderly patients treated in a CGA unit more satisfied with their hospital care than those treated in conventional acute medical care?. Patient Prefer Adherence. 2018;12: 233-240.

17. http://www.alliedacademies.org/articles/effectiveness-ofcomprehensive-geriatric-assessment-in-elderly-patients-withcoronary-heart-disease.pdf

18. https://ascopubs.org/doi/abs/10.1200/JCO.2017.35.31_suppl. 115

19. Fried L. Frailty in Older Adults: Evidence for a Phenotype. The J Gerontology. 2001;56; 146-157.

20. Yaman H. The Vulnerable Elders-13 Survey Predicts 5-year Functional Decline and Mortality Outcomes Among Older Ambulatory Care Patients. J Am Geriatr Soc. 2009;57: 2070-2076.

21. Yaman H, Ünal Z (2019) The validation of the PRISMA-7 questionnaire in community-dwelling elderly people living in Antalya, Turkey. Electron Physician. 2018;10: 7266-7272.

22. Dent E, Kowal P, Hoogendijk E. Frailty measurement in research and clinical practice: A review. Eur J Int Med. 2016;31: 3-10.

23. Metzelthin S, Daniëls R. Research article The psychometric properties of three self-report screening instruments for identifying frail older people in the community. BMC Public Health. 2010;10:176.

24. https://www.researchgate.net/publication/ 331215164_The_ability_of_four_frailty_screening_instruments_to _predict_mortality_hospitalization_and_dependency_in_instrume ntal_activities_of_daily_living

25. Rolfson D, Majumdar S. Validity and reliability of the Edmonton Frail Scale. Age Ageing. 2006;35:526-529.

26. https://www.acpinternist.org/weekly/archives/ 2013/11/19/6.htm.

27. Gobbens R (2019) Exploring the efficiency of the Tilburg Frailty Indicator: a review. Clinical Interventions in Aging. 2017;12: 1739-1752.

28. Perna S. Performance of Edmonton Frail Scale on frailty assessment: its association with multi-dimensional geriatric conditions assessed with specific screening tools. BMC Geriatr. 2017;17: 2 